\title{
Clinical and immunopathological aspects of disseminated cutaneous leishmaniasis
}

\author{
E.M. Carvalho ${ }^{\mathrm{a}, *}$, A. Barral ${ }^{\mathrm{a}}$, J.M.L. Costa ${ }^{\mathrm{b}}$, A. Bittencourt ${ }^{\mathrm{a}}$, \\ P. Marsden ${ }^{\mathrm{c}}$ \\ ${ }^{a}$ Serviço de Imunologia e Departamento de Patologia do Hospital Universitário Prof. Edgard Santos, \\ 40.110-160, Salvador, Brazil, ' Universidade Federal do Maranhão, Maranhão, Brazil, \\ ${ }^{\mathrm{c}}$ Universidade de Brasilia, Brasilia, Brazil
}

(Received 20 August 1993; revised 26 November 1993; accepted 10 December 1993)

\begin{abstract}
The clinico-pathological and immunological findings in eight patients from Bahia, Brazil with disseminated cutaneous leishmaniasis are described. This condition differs from anergic diffuse cutaneous leishmaniasis (DCL) and from classical American cutaneous leishmaniasis (ACL). The number of lesions in these patients ranged from 75 to 800 and were characterized by papules and an acneiform type of lesion with a few ulcers rather than nodules that are the main characteristic of DCL. On the other hand the high incidence of mucosal disease $(38 \%)$ in patients with disseminated cutaneous leishmaniasis make the prevalence of mucosal involvement in this condition higher than that observed in ACL. L. amazonensis (five cases) and $L$. braziliensis (two cases) were the causal agents in the patients where the infecting agent was characterized. Antibody titers in disseminated cutaneous leishmaniasis were higher than those observed in ACL and patients with the highest antibody titers had mucosal envolvement. Abnormalities in cellular immunity that are not observed in ACL such as decrease in $\mathrm{CD}_{4}+$ cells and absence of $T$ cell response to leishmania antigen were observed in several patients with disseminated cutaneous leishmaniasis but restoration of these abnormalities occurred after treatment. In spite of the great number of lesions, the therapeutic response was good in six patients with disappearance of the lesions in a period shorter than that observed in ACL. In the two patients that presented therapeutic failure the causal agent was $L$. amazonensis. In such patients there was a predominance of ulcerated lesions, and a high titer of antibody was detected.
\end{abstract}

Key words: Leishmaniasis; Cutaneous leishmaniasis; Disseminated cutaneous leishmaniasis; Tegumentary leishmaniasis; Immunology in leishmaniasis

\section{Introduction}

Disseminated cutaneous leishmaniasis is clinically characterized by the presence of multiple papular and ulcerated lesions. The differential diagnosis of this condition is made with the anergic type of disease called diffuse cutaneous leishmaniasis (Convit et al., 1972; Bittencourt and Guimarães, 1968; Bryceson, 1970). Patients with diffuse cutaneous leishmaniasis (DCL) have nodular disseminated lesions with heavily parasitized macrophages and relatively poor lymphocyte infiltration (Bryceson, 1970; Convit et al., 1972). Additionally patients with DCL lack a T cell

*Corresponding author. 
proliferative response and intradermal skin test reaction to leishmania antigens (Bryceson, 1970; Petersen, et al., 1982). Multiple cutaneous lesions due to leishmania infection have also been documented in post-Kala-azar dermal leishmaniasis (Haldar et al., 1983), and in a rare clinical presentation of cutaneous leishmaniasis called leishmanids (Berlin and Brenner, 1982). In the New World, patients with disseminated leishmania infection have been described (Costa et al., 1986), but the clinical picture, the pathology of this condition and host immune response during this infection has not been studied. Here we describe clinical, histopathological, and immunological findings in eight patients with disseminated cutaneous leishmaniasis, and indicate clear differences between this form of leishmania infection, DCL and classical American cutaneous leishmaniasis (ACL).

\section{Material and methods}

\section{Subjects}

The patients were seen at the Hospital Universitário Professor Edgard Santos in the state of Bahia, Brazil in the period from 1986 to 1992. Data of the clinical history and of a complete physical examination were obtained, and parasite culture, skin test and immunological studies were performed.

\section{Parasite isolation, cultivation and characterization}

The isolation was made by culture in tubes of NNN blood agar overlaid with a modified liver infusion tryptose from samples obtained by aspiration of the lesions. The culture from the biphasic medium were expanded for growth in modified LIT medium and kept at $25 \geqslant C$. Procedures for both serodeme analysis with monoclonal antibodies and enzyme electrophoresis have been reported previously (Grimaldi et al., 1987).

\section{Histopathological studies}

Skin biopsies were performed with a $4 \mathrm{~mm}$ Baker's biopsy punch (Baker/Cummins Pharmaceuticol Inc, Miami, Fl), after local anesthesia (1\% lidocaine), and fixed in buffered formol. Sections of paraffin embeded tissue were stained with hematoxylin-eosin.

\section{Immunological studies}

Immunological studies included evaluation of T cell surface markers, 'in vitro' test of lymphocyte proliferation, and determination of IFN- $\gamma$ in supernatants of cultures of peripheral blood mononuclear cells (PBMC) stimulated with leishmania lysate. PBMC were separated from heparinised venous blood by gradient centrifugation using Ficol Hypaque. For proliferation assays (Carvalho et al., 1985), PBMC were adjusted to $10^{6} / \mathrm{ml}$ in RPMI (Gibco, New Island, NY) containing penicillin and streptomycin and supplemented with $10 \% \mathrm{AB}$ normal human serum. Aliquots of $2 \times 10^{5}$ cells in $0.2 \mathrm{ml}$ were cultured in triplicate in flat-bottomed microtiter plates 
(Linbro Chemical, New Haven, CT) and stimulated with soluble $L$. amazonensis lysate $(5 \mu \mathrm{g} / \mathrm{ml})$, cell proliferation was measured by $\left[{ }^{3} \mathrm{H}\right]$ thymidine uptake after 5 days of incubation (Carvalho et al., 1985). The number of T cells, $\mathrm{CD}_{4}{ }^{+}$cells and $\mathrm{CD}_{8}{ }^{+}$cells were determined by reaction with monoclonal antibodies anti $\mathrm{OKT}_{11}$, $\mathrm{OKT}_{4}, \mathrm{OKT}_{8}$ (Ortho Diagnostic, Paritan, $\mathrm{NJ}$ ) followed by addition of a fluorescent rabbit anti-mouse immunoglobulin (Carvalho, et al., 1985). Determination of IFN- $\gamma$ production was evaluated in supernatants of lymphocyte cultures $\left(3 \times 10^{6}\right.$ cells $/ \mathrm{ml}$ stimulated with $20 \mu \mathrm{g}$ of crude Leishmania antigen). IFN- $\gamma$ levels were determined by a sandwich ELISA (Kemp et al., 1991).

\section{Antigen and serological test}

Leishmania promastigote lysate was prepared from a cloned isolate of $L$. amazonensis (MHOM BR86-BA125) from a patient with cutaneous leishmaniasis. Antigen used for skin test and $T$ cell studies were prepared as previously described (Carvalho et al., 1985; Reed et al., 1986). Serological tests were performed by indirect immunofluorescence using promastigotes of $L$. amazonensis (Badaró et al., 1983).

\section{Results}

The clinical picture of the cases presented includes the presence of papules and acneiform lesions ranging in number from 75 to more than 800 and a few ulcers (Fig. 1). These lesions were disseminated and present on the face, chest, abdomen, and limbs. The clinical profile, antibody titer to leishmania antigen and therapeutic response in these patients are shown in Table 1 . All patients were adult males with an age range of 23 to 62 years. Fever, chills and malaise were complaints of six patients in the initial phase of illness (before or in the first week after the appearance of the dissemination). In four individuals reference was made to an initial lesion in one leg followed by dissemination to the entire body after a period as short as two days. In only one case the initial lesion had a long duration (six months) before dissemination. Mucosal lesions were detected in two patients in the initial examination (patients no. 5, no. 7). A third patient (no. 4) developed mucosal disease during follow-up. Leishmania was isolated in culture in seven patients and L. amazonensis was the causal agent in five of them. Anti-leishmania antibody was documented in all patients with titers ranging from 1:64 to 1:1024. In three patients who had mucosal involvement, antibody titers were higher than 1:512 and two of them failed to respond to antimonial therapy. The response to antimonial therapy was prompt in the other six patients. Five of this patients were completely free of active lesions in a period of two months. In such cases a scar with minimal skin retraction was documented after cure. In one of these six patients who respond to antimonial therapy mucosal involvement was documented at initial evaluation, but the patient was free of cutaneous and mucosal involvement, 30 and 90 days respectively after initiation of therapy.

In five of the six patients who were cured, two series of antimonial therapy were given ( $20 \mathrm{mg} \mathrm{Sb} / \mathrm{kg} /$ day for 20 days), with an interval of 10 days between the series, and in one patient only one course of antimonial therapy was used with disappearance of the lesions in thirty days. In two patients (no. 4, no. 5) there was only 


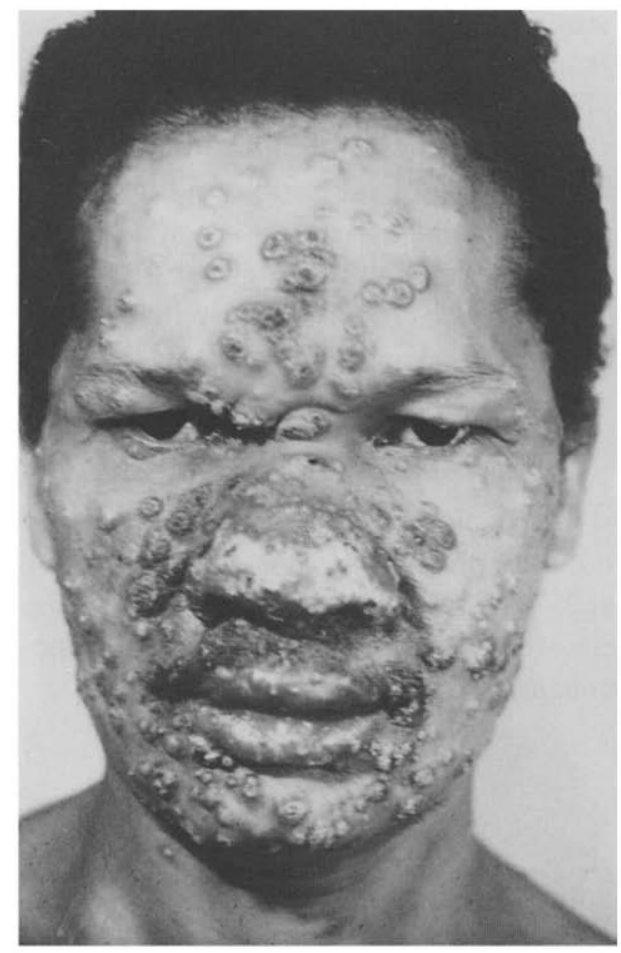

(a)

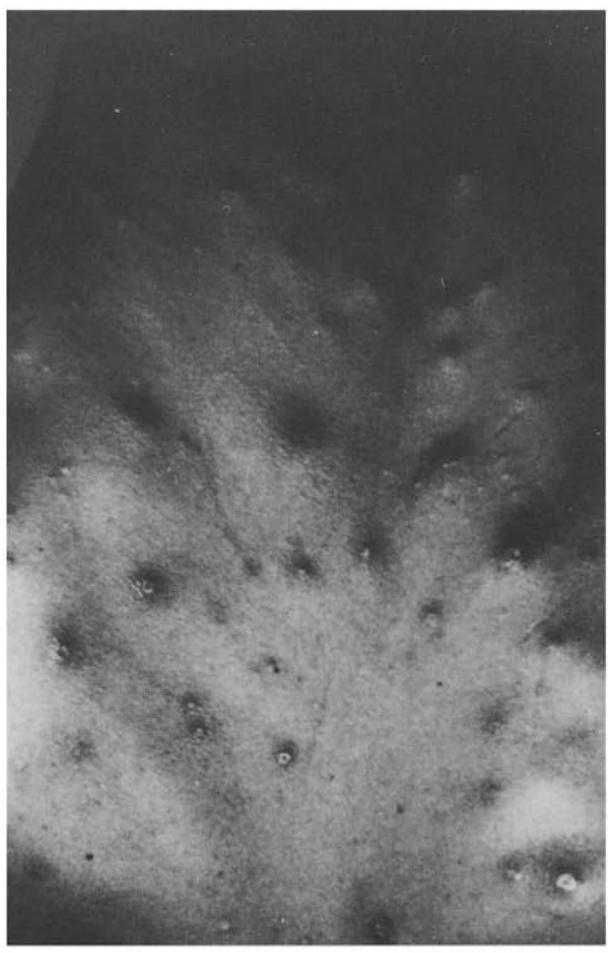

(b)

Fig. 1. (a) Numerous acneiform lesions in the face. In the nose a diffuse infiltration with small ulcer is observed. (b) See pustules of different sizes surrounded by an erythematous halo (acneiform lesions) on the chest.

partial response with healing of the majority of the lesions but with persistence of a few ulcers and reactivation of the disease in healed areas upon cessation of therapy. One of these two patients had mucosal disease in the initial evaluation. In the other patient in addition to skin lesions a mucosal disease was detected 6 months after the initiation of the treatment.

The histopathological studies (Table 2) revealed a mononuclear infiltrate with predominance of plasma cells. Granulomatous reaction and parasitism were observed in two patients. Only one case presented with pseudocarcinomatous hyperplasia. Biopsies of the acneiform lesions were performed in three patients and all showed a follicular pattern. A heavy inflammatory reaction was observed around the follicles. The involvement was predominant in the follicular infundibulum (Fig. 2a) although all the follicule may be compromised (Fig. 2b). A partial disruption of the follicular epithelial sheath with marked spongiosis and exocytosis was observed associated with intrafollicular plugging (Fig. 2a). In one acneiform lesion of very recent onset (three days of appearance) a marked infiltration of polymorphonuclear cells with predominance eosinophils was observed around one follicule (case 4).

Data of the skin test, leishmania antigen specific lymphocyte proliferative response and circulating $T$ cells subsets are also shown in Table 2 . Delayed type hypersensitivity to leishmania antigen was absent in four patients (nos. 2,3,5,6). Absence of lymphocyte proliferation to leishmania lysate was documented in three of the four 


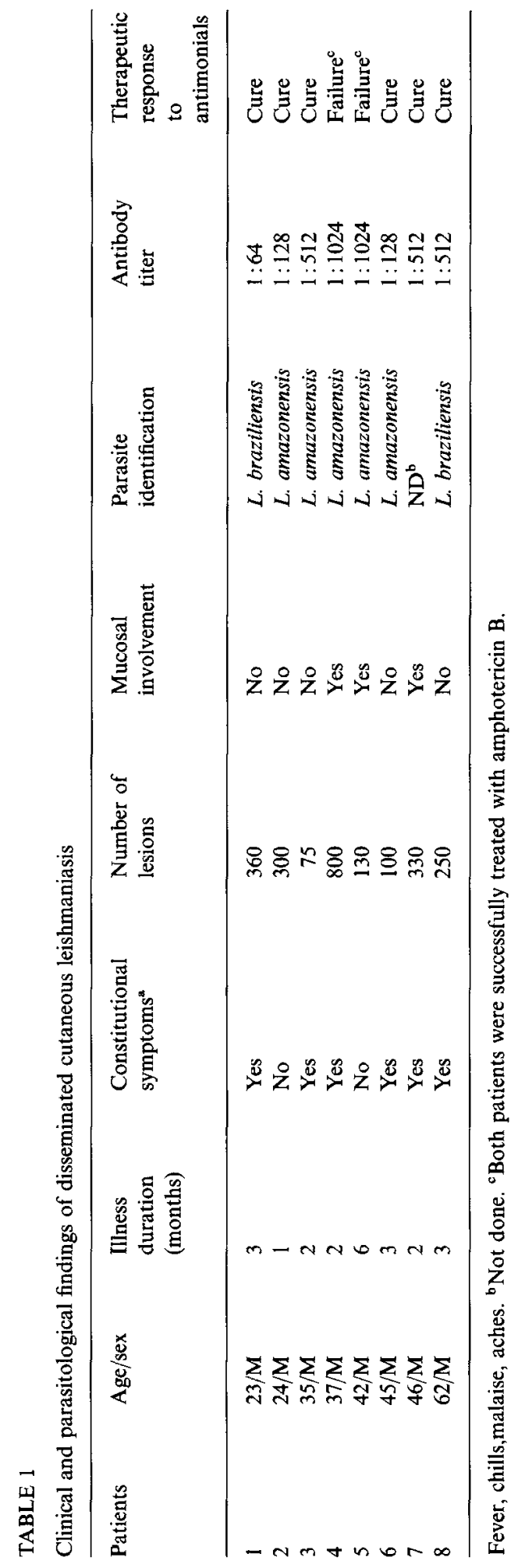




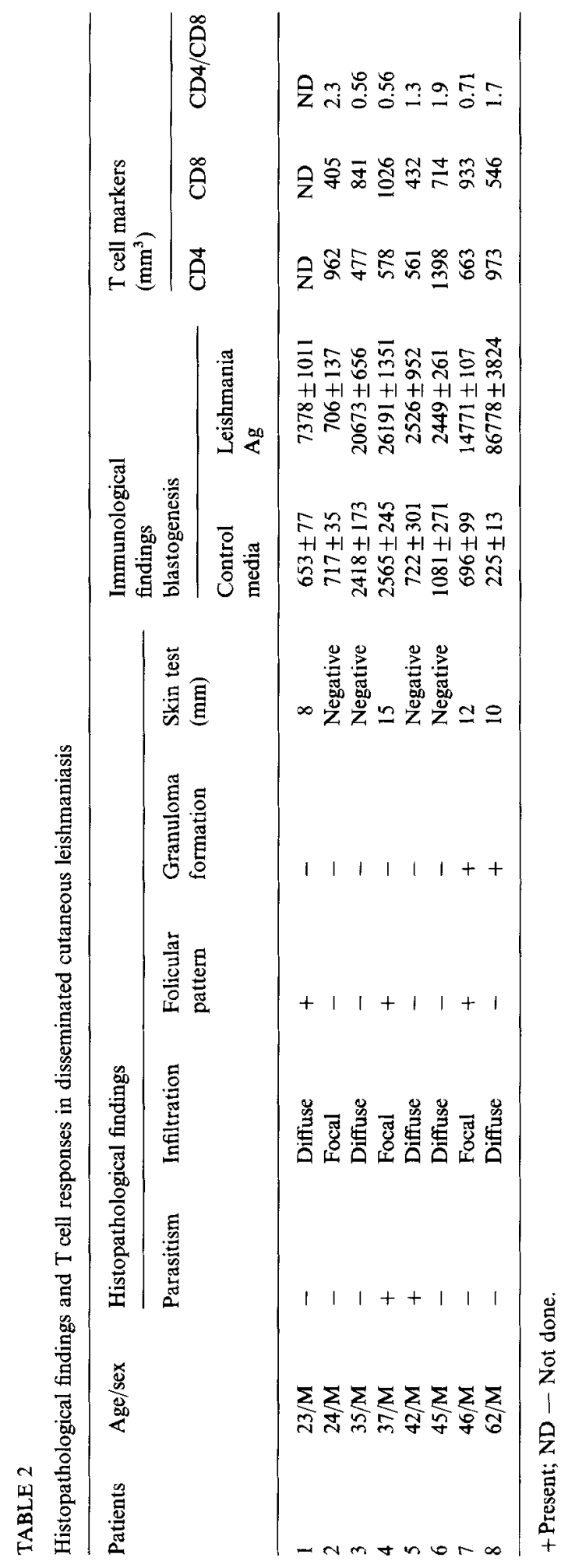




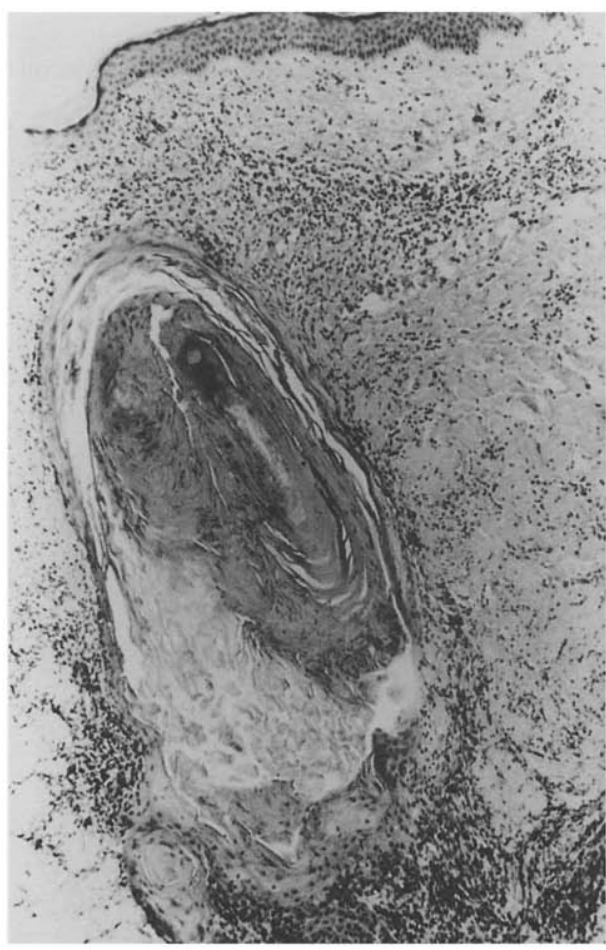

(a)

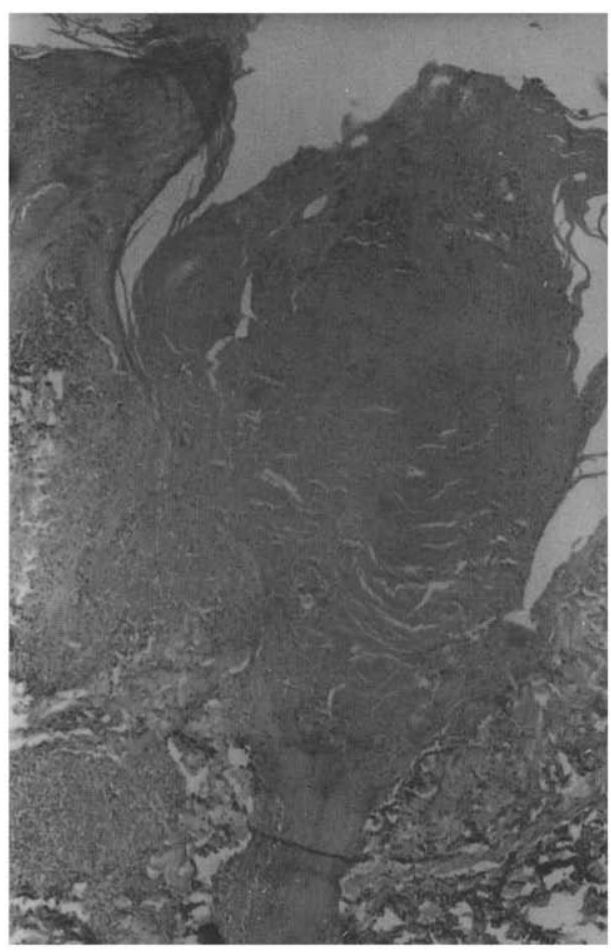

(b)

Fig. 2. (a) A longitudinal section of a hair follicle shows a marked dilatation with plugging of the infundibulum. A partial disruption of the follicular epithelial sheath and a chronic inflamatory infiltrate can also be observed. HE, $\times 64$. (b) An oblique section showing dilatation and plugging of the whole hair follicle with atrophy of the follicular epithelial sheath. A chronic inflammatory infiltrate is seen around the follicle. HE, $\times 64$.

patients with negative skin test (nos. 2,5,6). In all cases lacking $T$ cell response to leishmania antigen (absence of lymphocyte proliferation and/or negative skin test) the infecting agent was $L$. amazonensis. No correlation between the degree of $\mathrm{T}$ cell proliferation to leishmania antigens and the therapeutic response could be documented. Of the three patients whose lymphocytes did not proliferate to leishmania lysate only one did not respond to antimonial therapy. In the three patients that had negative lymphocyte proliferative responses during active disease, restoration of this response was observed in subsequent evaluations after cure in two patients and in a third patient after resolution of the majority of the lesions. Low counts of $\mathrm{CD}_{4}+$ cells (less than mean minus one standard of controls $\neq 694$ cells $\mathrm{mm}^{3}$ ) was observed in four patients (nos. 3,4,5,7). In three of them L. amazonensis was the causal agent. The two patients who failed to antimonial therapy had low CD4 counts. $\mathrm{CD}_{4}+$ cells observed in these patients was however much higher than one observes in AIDS patients, and HIV antibodies were absent in all cases with disseminated cutaneous leishmaniasis. In two patients (nos. 4,7) IFN- $\gamma$ levels were determined in supernatant of lymphocyte culture stimulated with PHA or leishmanial antigen. High levels of IFN- $\gamma$ were detected $(456 \mathrm{pg} / \mathrm{ml}$ and $394 \mathrm{pg} / \mathrm{ml}$ respectively) in 
TABLE 3

Clinical and immunopathological findings in disseminated cutaneous leishmaniasis, diffuse cutaneous leishmaniasis and classical American cutaneous leishmaniasis

\begin{tabular}{|c|c|c|c|}
\hline \multirow{2}{*}{$\begin{array}{l}\text { Clinical and immuno- } \\
\text { pathological findings }\end{array}$} & \multicolumn{3}{|c|}{ Clinical forms of leishmaniasis } \\
\hline & Disseminated & DCL & ACL \\
\hline Clinical presentation & $\begin{array}{l}\text { papules and acneiform } \\
\text { lesions }\end{array}$ & nodules & ulcers \\
\hline Number of lesions & many & many & one or few \\
\hline Destructive mucosal involvement & frequent & absent & infrequent \\
\hline Parasite identification & $\begin{array}{l}\text { L. amazonensis } \\
\text { L. braziliensis }\end{array}$ & L. amazonensis & $\begin{array}{l}\text { L. amazonensis } \\
\text { L. braziliensis }\end{array}$ \\
\hline Parasites in tissue & rare or absent & abundant & rare or absent \\
\hline Follicular pattern & present & absent & absent \\
\hline Granulomatous pattern & present or absent & absent & present or absent \\
\hline Intradermal skin test & positive or negative & negative & positive \\
\hline Antibody titers & mainly high & high & low \\
\hline Lymphocyte proliferative response & present or absent & absent & present \\
\hline
\end{tabular}

cultures stimulated with PHA and $(366 \mathrm{pg} / \mathrm{ml}$ and $420 \mathrm{pg} / \mathrm{ml})$ in supernatants from cultures stimulated by leishmanial antigen.

A comparison between the clinical, histopathological and immunological findings of the disseminated cutaneous leishmaniasis with the DCL and the classical ACL is in Table 3. In disseminated cutaneous leishmaniasis multiple acneiform lesions are the main clinical findings. The causal agent is predominantly $L$. amazonensis and a follicular pattern is found in the histopathological studies. The absence of lymphocyte proliferative response to leishmania antigens and absence of DTH seen in disseminated cutaneous leishmaniasis is transitory and such patients can produce IFN- $\gamma$.

\section{Discussion}

ACL in Brazil is predominantly caused by $L$. braziliensis and the most frequent clinical form of presentation is a single ulcer in the leg. (Llanos-Cuentas et al., 1984). Immunological studies in ACL have documented a preserved $T$ cell response to leishmania antigen and the ability of macrophages to kill intracellular amastigotes (Carvalho et al., 1985; Conceição-Silva et al., 1990). At the other pole of the disease is the diffuse cutaneous leishmaniasis characterized by multiple nodules and absence of T cell response to leishmania antigen (Bittencourt and Guimarães, 1968; Peterscn et al., 1982). In Brasil all cases of DCL have been associated to $L$. amazonensis infection (Lainson, 1983; Barral et al., 1991). The cases described here of disseminated cutaneous leishmaniasis have clinical immunological and therapeutic aspects that clearly differentiate this condition from the DCL and from the classical ACL. In disseminated cutaneous leishmaniasis the main findings are the predominance of papules and acneiform type of lesions associated less frequently with ulcers. Papules are rarely observed and acneiform type of lesion are not seen in classical ACL. Otherwise, in DCL the lesion are predominantly nodular. Additionally in the present series a high frequency of mucosal disease was observed while the prevalence of 
mucosal involvement in the endemic areas of cutaneous leishmaniasis is less than $5 \%$ (Jones et al., 1987). Clinically disseminated cutaneous leishmaniasis resemble the rare form of leishmaniasis reported in the old world called leishmanid that is defined as an eruption of papules and pustules that appears in a different site from the primary focus of leishmaniasis (Berlin and Brenner, 1982). The word leishmanid was used to indicate a hypersensitivity reaction since delayed hypersensitivity was present in the few cases reported (Berlin, 1953) and histopathologically a tuberculoid reaction was observed. The present study show that immunological responses in disseminated cutaneous leishmaniasis are quite variable and both a strong $\mathrm{T}$ cell response or absent cell mediated immunity may be present.

The histopathological studies in all of these patients revealed the same histological pattern observed in New World cutaneous leishmaniasis represented by a mononuclear cell infiltration with predominance of plasma cells associated with granulomatous reaction (Bittencourt and Barral, 1991). Different from the classical ACL was the low frequency of epidermal hyperplasia and the occurrence of a follicular pattern in the acneiform lesion. Considering that acneiform lesions were observed in all cases except one, it is probably that circulating parasites present a preference for the follicular area. It is possible that a follicular pattern was not observed in all the cases due to a complete obliteration of the follicular structure by the inflammatory reaction in older lesions. As observed in classical ACL (Bittencourt and Andrade, 1967) a tendence to destroy the cutaneous appendages was observed. Occurrence of a more pronounced eosinophil infiltrate, as observed in a recent acneiform lesion, is also referred in the initial lesions in experimental leishmaniasis (Pompeu et al., 1992).

The immunological studies in patients with disseminated cutaneous leishmaniasis are different from those observed in ACL and in DCL. For instance depression of the cellular immunity was observed in several patients, but restoration of $T$ cell response to parasite antigen could be documented after the cure or improvement of the disease. Patients with disseminated cutaneous leishmaniasis may also exibit preserved $\mathrm{T}$ cell reponses to leishmania antigen. The antibody titers to leishmania antigen in this form of leishmaniasis were higher than that observed in classical ACL.

Although the mechanism of dissemination of the disease is not clear, the rapid spread of the infection, absence of lymph node enlargment and the occurrence of general symptoms such as fever, chills and malaise suggest direct heamatogenous dissemination. For such dissemination, factors related to the nature of the parasite and to the host may be involved. Different from the classical ACL in Brazil where the causal agent is predominantly L. braziliensis (Grimaldi ct al., 1987) and a preserved $\mathrm{T}$ cell response to leishmania antigen is observed (Carvalho et al., 1985) most of the patients with disseminated leishmaniasis were infected by $L$. amazonensis and variable degree of abnormalitites in $T$ cell response could be documented. Therefore an association of factors related to the parasite with alteration of the immune response of the host may be involved in dissemination of the infection.

The therapeutic response to pentavalent antimonials in disseminated cutaneous leishmaniasis was quite variable. Several patients with hundreds of lesions could be free of symptoms and without scars as soon as after two months of initiation of therapy, and no evidence of cutaneous or mucosal disease was observed after a follow-up from 4 to 7 years in five of such patients. In two cases therapeutic failure was documented. The failure of response to antimonial therapy was associated with 
L. amazonensis infection, high antibody titer, mucosal involvement and a low CD4 count.

\section{Acknowlegments}

This study was supported by Grant AI30639 from the National Institutes of Health and UNDP World Bank/WHO Special Program for Research and Training in Tropical Diseases and CNPq.

\section{References}

Badaró, R., Reed, and Carvalho, E.M. (1983) Immunofluorescent antibody test in American visceral leishmaniasis: sensitivity and specificity of different morphological forms of two leishmania species. Am. J. Trop. Med. Hyg. 32, 480-484.

Barral, A., Pedral-Sampaio, D., Grimaldi, G., Momem, H., McMahon-Pratt, D., Ribeiro de Jesus, A., Almeida, R., Badaró, R., Barral-Netto, M., Carvalho, E.M. and Johnson, W. (1991) Leishmaniasis in Bahia, Brazil: evidence that Leishmania amazonensis produces a wide spectrum of clinical disease. Am. J. Trop. Med. Hyg. 44, 536-546.

Berlin, C. (1953) Leishmanid: report of case. Br. J. Dermatol. 65, 265-268.

Berlin, C. and Brenner, S. (1982) Leishmanid. Isr. J. Med. Sci. 18, 285-286.

Bittencourt, A.L. and Andrade, Z.A. (1967) Aspectos imunopatológicos na leishmaniose cutâneo-mucosa. Hospital (Rio), 71, 975-984.

Bittencourt, A.L. and Guimarães, N. (1968) Imunopatologia da leishmaniose tegumentar difusa. Med. Cutan. Ibero Lat. Am. 2, 395-402.

Bittencourt, A.L. and Barral, A. (1991) Evaluation of the histopathological classifications of american cutaneous and mucocutaneous leishmaniasis. Memór. Inst. O. Cruz 86, 51-56.

Bryceson, A.D.M. (1970) Diffuse cutaneous leishmaniasis in Ethiopia. Immunological studies and pathogenesis. Trans. R. Soc. Trop. Med. Hyg., 64, 380-387.

Carvalho, E.M., Johnson, W.D., Barreto, E., Marsden, P.D., Costa, J,. M., Reed, S.G. and Rocha, H. (1985) Cell mediated immunity in American cutaneous and mucosal leishmaniasis. J. Immunol. 135, 4144-4148.

Conceição Silva, F., Dórea, R.C., Pirmez, C., Schubach, A. and Coutinho, S.G. (1990) Quantitative study of $L$. braziliensis reactive T cells in peripheral blood and in the lesions of patients with American mucocutaneous leishmaniasis. Clinical Exp. Immunol. 79, 221-226.

Convit, J., Pinardi, M.E. and Rondon, A.J. (1972) Diffuse cutaneous leishmaniasis: A disease due to an immunological defect of the host. Trans. R. Soc. Trop. Med. Hyg. 66, 603-610.

Costa, J.L.M., Marsden, P.D., Llanos-Cuentas, E.A., Netto, E.M., Carvalho, E.M., Barral, A., Rosa, A.C., Cuba, C.C., Magalhães, A.V. and Barreto, A.C. (1986) Disseminated cutaneous leishmaniasis in a field clinic in Bahia, Brazil: a report of eight cases. J. Trop. Med. Hyg. 89, 319-323.

Grimaldi, G., David, J.R. and McMahon-Pratt, D. (1987) Identification of New World Leishmania species characterized by serodeme analysis using monoclonal antibodies. Am. J. Trop. Med. Hyg. $36,270-287$.

Haldar, J.P., Chose, S., Saha, K.C. and Chose, A.C. (1983) Cell mediated immunity in Indian kala-azar and post kala-azar dermal leishmaniasis. Infect. Immun. 42, 702-707.

Jones, T.C., Johnson, Jr. W.D., Barreto, A., Badaró, R., Cerf, B., Reed, S.G., Netto, E.M., Tada, M.S., França, F., Weise, W., Golightly, L., Fikrik, E., Costa, J.M.M., Cuba, C,. C., Marsden, P.D. (1987) Epidemiology of American cutaneous leishmaniasis due to L. braziliensis braziliensis. J. Infect. Dis. $156,73-83$.

Kemp, M., Theander, T.G., Handman, E., Hey, A.S., Kurtzhals, J.A., Huiid, L., Sorensen, A.L., Were, J.O.B., Roech, D.K. and Kharazmi, A. (1991) Activation of human T lymphocytes by leishmania lipophosphoglycans. Am. J. Trop. Med. Hyg. 33, 219-224. 
Lainson, R. (1983) The American leishmaniasis: some observations on their ecology and epidemiology. Trans. R. Soc. Trop. Med. Hyg. 77, 569-596.

Llanos-Cuentas, E.A., Marsden, P.D., Lago, E.L., Barreto, A.C., Cuba, C.C. and Johnson, W.D. (1984) Human mucocutaneous leishmaniasis in Três Braços, Bahia-Brazil. An area of L. braziliensis braziliensis transmission. II Cutaneous disease: Presentation and evolution. Rev. Soc. Brasil. Med. Trop. 17, 169-177.

Petersen, E.A., Neva, F.A., Oster, C.N. and Diaz, H.B. (1982) Specific inhibition of lymphocyteproliferation responses by adherent suppressor cells in diffuse cutaneous leishmaniasis. N. Engl. J. Med. 306, 387-391.

Pompeu, M.L., Freitas, L.A., Santos, M.L.V., Barral, A. and Barral-Netto, M. (1992) Leishmania amazonensis infection: A comparison of in vivo leishmanicidal mechanisms between immunized and naive infected BALB/c mice. Exp. Parasitol., 74, 169-176.

Reed, S.G., Badaró, R., Masur, H., Carvalho, E.M., Lorenço, R., Lisboa, A., Teixeira, R., Johnson, W.D. and Jones, T.C. (1986) Selection of a specific skin test antigen for American visceral leishmaniasis. Am. J. Trop. Med. Hyg. 35, 79-85. 